\title{
Resistance against beet armyworms and cotton aphids in caffeine-producing transgenic chrysanthemum
}

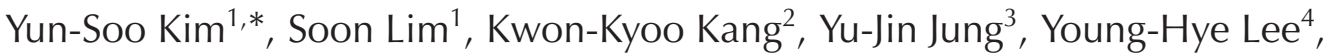 \\ Yong-Eui Choi ${ }^{1}$, Hiroshi Sano ${ }^{1,5}$ \\ ${ }^{1}$ Department of Forest Resources, College of Forest and Environmental Sciences, Kangwon National University, \\ Chuncheon 200-701, Korea; ${ }^{2}$ Department of Horticulture, Hankyong National University, Ansung 456-749, Korea; \\ ${ }^{3}$ Institute of Genetic Engineering, Hankyong National University, Ansung 456-749, Korea; ${ }^{4}$ Yesan Chrysanthemum, \\ Chungnam Provincial A.R.E.S., Yesan 340-915, Korea; ${ }^{5}$ Department of Botany, Stockholm University, 10691 \\ Stockholm, Sweden \\ *E-mail: yskim71@gmail.com Tel \& FAX: +82-33-250-8310
}

Received April 1, 2011; accepted May 10, 2011 (Edited by T. Aoki)

\begin{abstract}
Transgenic chrysanthemum plants were constructed to simultaneously express three $N$-methyltransferases involved in caffeine biosynthetic pathways. Resulting plants produced caffeine at approximately $3 \mu \mathrm{gg}^{-1}$ fresh tissue, and were tested for herbivore repellence. When starved second-instar caterpillars of beet armyworms (Spodoptera exigu) were allowed to feed, they ate up to $4.4 \mathrm{~mm}^{2}$ of leaf discs from the wild type plants, while less than $1.5 \mathrm{~mm}^{2}$ of those from the transgenic plants. When third-instars of cotton aphid (Aphis gossypii) were subjected to a choice-test, 27 gathered on wild type leaves, and 6 on transgenic leaves. These results indicate that caffeine-producing chrysanthemum is resistant against herbivores, lepidoptera caterpillars and aphids, both being one of the most serious pests in agriculture. We propose that the method can be practically applied to a variety of important plant species to confer resistance against biotic stresses.
\end{abstract}

Key words: Aphis gossypii, caffeine, chemical defense, chrysanthemum, Spodoptera exigu.

Plants cope with a variety of biotic stresses by producing secondary metabolites, which exhibit detrimental effects on living organisms (Dixon 2001; Dangl and Jones 2001; Field et al. 2006). The mechanism is referred as chemical defense. Caffeine (1,3,7-trimethylxanthine) is a typical secondary metabolite belonging to the purine alkaloid family, and considered to be a representative example of chemical defense (Ashihara et al. 2008). Recent studies showed that transgenic tobacco plants producing caffeine were resistant against tobacco cutworms (Spodoptera litura) and pathogenic microbes including Pseudomonas syringe and tobacco mosaic virus (Kim and Sano 2008; Kim et al. 2010; Uefuji et al. 2005). These findings suggested that agriculturally important crops could be improved by endogenously producing caffeine to confer resistance against a broad range of biotic tresses including diseases and herbivorous pests.

Chrysanthemum (Chrysanthemum morifolium) is one of the economically important ornamental plants (Shinoyama et al. 2002; Teixeira da Silva 2003). Breeders have long been attempting to create new and attractive varieties, which are uniform and reliable in production, easy to handle in a partly mechanized production system, and resistant to pests and diseases (Visser et al. 2007). The latter is particularly critical as the plant is susceptible to a broad array of herbivorous insects including leafminers, aphids, and beet armyworms (Visser et al. 2007). Many of these pests rapidly develop tolerance against insecticides, and development of efficient counteractions in breeding program has urgently and continuously been required. One such method could be construction of transgenic plants, which produce anti-herbivore substance(s). We previously reported that transgenic chrysanthemum producing caffeine exhibited a high level of salicylates and a strong resistance against pathogenic fungus, Botrytis cinerea (Kim et al. 2011). Here we describe that such transgenic chrysanthemum plants are also resistant against herbivores, beet armyworms and cotton aphids, which are the most serious pests in breeding.

Fifty leaf discs prepared from Chrysanthemum (Chrysanthemum morifolium cv. Shinba) plantlets grown under axenic conditions were transformed by Agrobacterium tumefaciens strain LBA4404 (Hiei et al. 1994) harboring the pBIN-NMT777, a multi-gene 
expression vector containing the three coffee $\mathrm{N}$ methyltransferases genes (CaXMT1, CaMXMT1 and CaDXMTI) and two selection marker genes (NPT II and $H P T$ ) (Uefuji et al. 2005). Leaf discs (ca. $1 \mathrm{~cm}^{2}$ ) were immersed with a bacterial suspension having $\mathrm{OD}_{600}$ value of 0.5 for $30 \mathrm{~min}$ in MS liquid medium (Murashige and Skoog 1962) containing 3\% sucrose and $50 \mathrm{mgl}^{-1}$ acetosyringone. After eliminating the bacterial suspension, the infected tissues were transferred to MS media containing $1.0 \mathrm{mgl}^{-1}$ 6-benzylaminopurine, $2.0 \mathrm{mg}^{-1}$ 1-naphthylacetic acid, $250 \mathrm{mg}^{-1}$ cefotaxime, and $20 \mathrm{mg}^{-1}$ kanamycin. After 1.5 months of the infection, calli and shoot primordia were simultaneously emerged from leaf discs cultured on medium containing $20 \mathrm{mg} \mathrm{l}^{-1}$ kanamycin and $250 \mathrm{mg}^{-1}$ cefotaxime (Figure
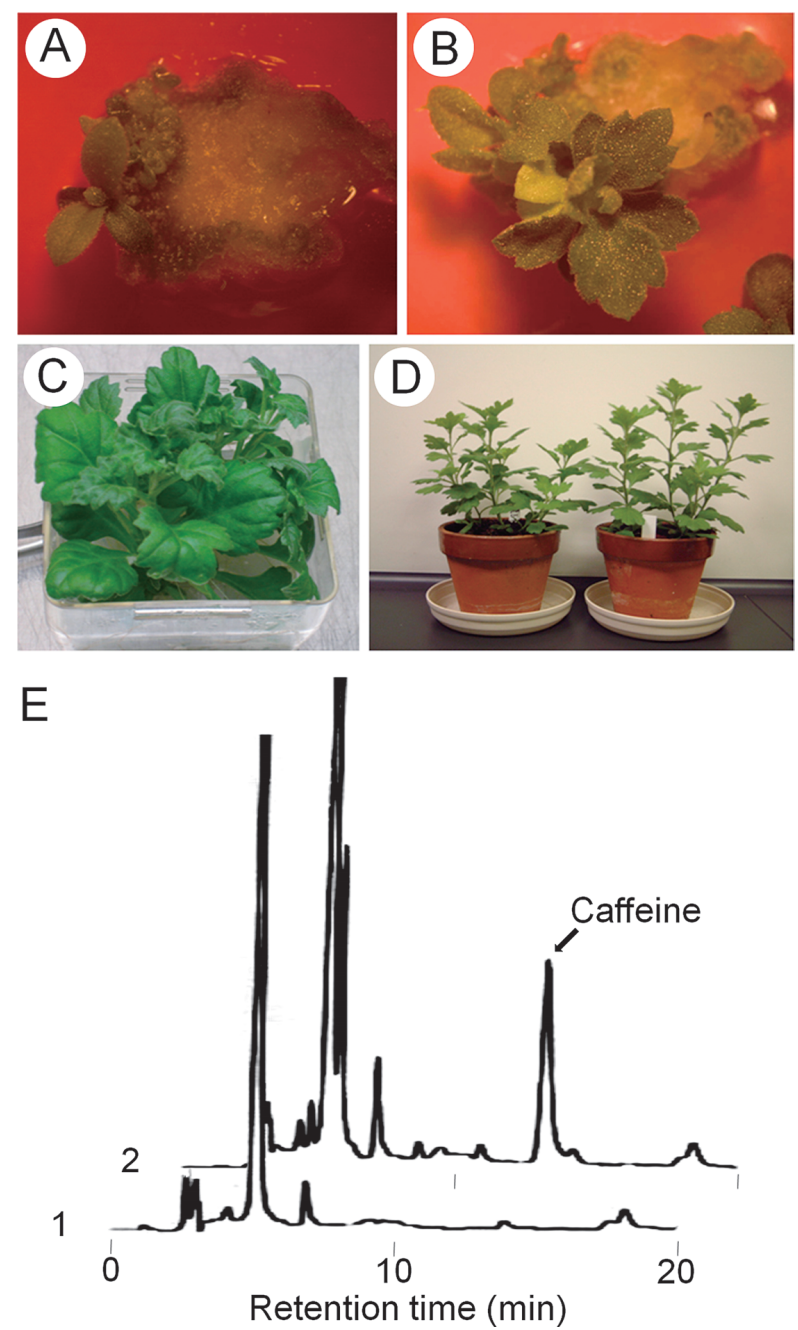

Figure 1. Transgenic chrysanthemum producing caffeine. (A) Induction of callus and shoot primordia from a leaf disc 1.5 months after Agrobacterium infection. (B) Stable transformants selected in MS medium containing kanamycin and cefotaxime. (C) Kanamycinresistant chrysanthemum plantlets ca. 3 months after antibiotic selection. (D) Chrysanthemum plants of a wild type (left) and a transgenic line $\mathrm{C \# 1}$ (right) grown in soil for about 2 months. (E) Detection of caffeine by HPLC in wild type (1) and transgenic chrysanthemum (2) in mature leaves.

1A). The candidates were subcultured onto MS medium containing kanamycin and cefotaxime with 3 weekintervals for selection of stable transformants (Figure 1B). Eight kanamycin-resistant transgenic shoots were obtained after about 3 months of antibiotic selection.

A
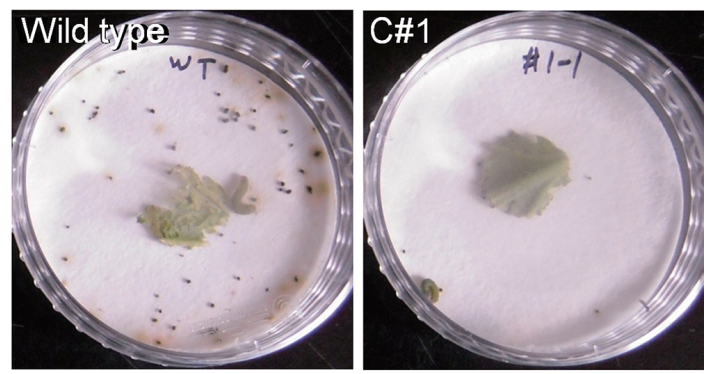

B
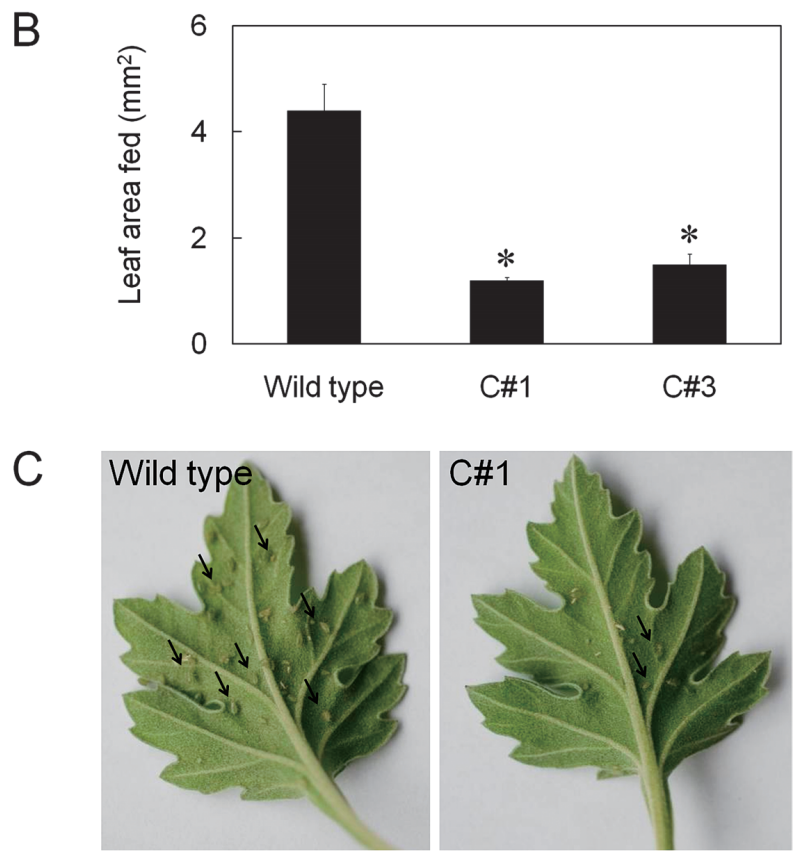

D

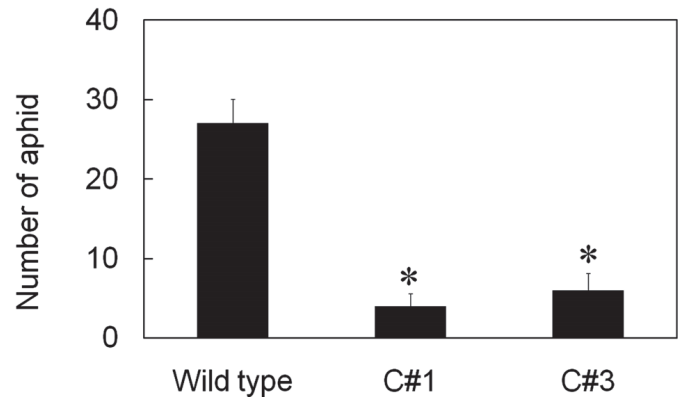

Figure 2. Insect bioassays. A non-choice test of beet armyworms (A, B). (A) After feeding for $24 \mathrm{~h}$ in the dark, each petridish containing wild type and transgenic leaf disc was photographed. (B) Feeding behavior was quantified by six replicate tests and fed leaf areas were calculated with the aid of a leaf area meter. A choice test of aphids $(\mathrm{C}$, D). (C) After feeding for a week, each leaf detached from whole plants of wild type or transgenic line C\#1 was photographed. (D) Feeding behavior of aphids was quantified by counting the number of aphids on 10 detached leaves with three replicates. Asterisks * indicate the significant differences between means determined with one-way ANOVA followed by Turkey's test $(P<0.05)$. 
They were transferred on hormone-free MS medium containing $10 \mathrm{mgl}^{-1}$ kanamycin for rooting. After 1 month of the culture, six transgenic plantlets rooted vigorously were selected (Figure 1C) and transferred to soil (Figure 1D). All six lines successfully expressed three transgenes and produced caffeiene at ca. $3 \mu \mathrm{g}$ perg fresh weight (Figure 1E shows a representative pattern of HPLC assay) (Kim et al. 2011). Two lines (C\#1 and C\#3) were selected, grown to the maturity and subjected to the insect bioassay.

Insect repellence was examined through feeding behaviors of beet armyworms (Spodoptera exigu) and cotton aphids (Aphis gossypii). For beet armyworm larva, the non-choice test was designed. Second-instar caterpillars were starved for 5 hours. Each caterpillar was transferred to each leaf disc (diameter $15 \mathrm{~mm}$ ) prepared from wild type or transgenic lines and then allowed to feed for $24 \mathrm{~h}$ in the dark in a climate chamber at $25^{\circ} \mathrm{C}$ with a relative humidity of $60 \%$. Total sample numbers were 54 in six independent assays. Digital photographs of the leaf discs were taken at the end point of feeding time, and then leaf areas consumed were calculated by a leaf area meter (LI-3100, Li-cor, USA). The results showed that caterpillars vigorously fed on wild type leaves, while they positively avoided transgenic leaves (Figure 2A). The repellency was numerically estimated by quantitative measurement of the eaten areas. On the average, caterpillars ate up to $4.4 \mathrm{~mm}^{2}$ of wild type leaf discs, while less than $1.5 \mathrm{~mm}^{2}$ of the transgenic ones (Figure 2B). For aphids, a choice test was designed. Clonal replicates of wild type and transgenic plants were produced from stem cuttings, transferred into vinyl pots (diameter $10 \mathrm{~cm}$, height $15 \mathrm{~cm}$ ), and grown in $30 \times 30 \times 40 \mathrm{~cm}$ transparent plastic cages within the controlled growth chamber. When the plants were ca. $20 \mathrm{~cm}$ tall, ten 3-instar aphids were applied in the cages for a week. At least three independent assays were performed. The results showed that aphids preferred the wild type leaves over the transgenic leaves (Figure 2C). A quantitative measurement indicated that the number of aphids were up to 27 for wild type, while less than 6 for the transgenic leaves (Figure 2D).

This paper is the fifth report on the transgenic plants which endogenously produce caffeine (Kim et al. 2006; Kim et al. 2008; Kim et al. 2011; Uefuji et al. 2005). In the first three reports, we handled tobacco plants as a model system, and showed a stable production of caffeine in mature plants, and resistance against biotic stresses, diseases and herbivores (Kim et al. 2006; Kim et al. 2008; Uefuji et al. 2005). In the forth report, we practically applied the method to chrysanthemum, which showed elevated salicylate levels together with resistance against fungus disease (Kim et al. 2011). In this note, we showed that the same transgenic chrysanthemum is resistant against multiple herbivore pests. The finding is significant as the plants are resistant not only against lepidoptera (moth), but also against aphidae, the most serious pests in the culture field. Taken together, we conclude that caffeine-producing transgenic plants are commonly resistant against a broad range of herbivore insects and pathogens, and that the method is widely applicable to agriculturally and economically important plant species. Currently we are attempting to construct transgenic rice plants, which will be of help to stably produce food in the future.

\section{Acknowledgements}

This work was supported by a grant from the World Class University Project of Korea.

\section{References}

Ashihara H, Sano H, Crozier A (2008) Caffeine and related purine alkaloids: biosynthesis, catabolism, function and genetic engineering. Phytochemistry 69: 841-856

Dangl JL, Jones JDG (2001) Plant pathogens and integrated defense responses to infection. Nature 411: 826-833

Dixon RA (2001) Natural products and plant disease resistance. Nature 411: 943-947

Field B, Jordan F, Osbourn A (2006) First encounters-development of defense-related natural products by plants. New Phytologist 172: 193-207

Hiei Y, Ohta S, Komari T, Kumahsiro T (1994) Efficient transformation of rice (Oryza sativa L.) mediated by Agrobacterium and sequence analysis of the boundaries of the T-DNA. Plant J 6: 271-282

Kim YS, Sano H (2008) Pathogen resistance of transgenic tobacco plants producing caffeine. Phytochemistry 69: 882-888

Kim YS, Choi YE, Sano H (2010) Stimulation of defense system by caffeine production in planta. Plant Signal Behav 5: 489493

Kim YS, Uefuji H, Ogita S, Sano H (2006) Transgenic tobacco plants producing caffeine: a potential new strategy for insect pest control. Transgenic Res 15: 667-672

Kim YS, Lim S, Yoda H, Choi CS, Choi YE, Sano H (2011) Simultaneous activation of salicylate production and fungal resistance in transgenic Chrysanthemum producing caffeine. Plant Signal Bahav 6: 409-412

Murashige T, Skoog F (1962) A revised medium for rapid growth and bioassays with tobacco tissue cultures. Physiol Plant 15: 473-497

Shinoyama H, Kazuma T, Komano M, Nomura Y, Tsuchiya T (2002) An efficient transformation system in chrysanthemum [Dendranthema $\times$ grandiflorum (Ramat.) Kitamura] for stable and non-chimeric expression of foreign genes. Plant Biotechnol 19: 335-343

Teixeira da Silva JA (2003) Chrysanthemum: advances in tissue culture, cryopreservation, postharvest technology, genetics and transgenic biotechnology. Biotechnol Adv 21: 715-766

Uefuji H, Tatsumi Y, Morimoto M, Kaothien-Nakayama P, Ogita S, Sano H (2005) Caffeine production in tobacco plants by simultaneous expression of three coffee $N$-methyltransferases and its potential as a pest repellant. Plant Mol Biol 59: 221227

Visser PB, de Maagd RA, Jongsma MA (2007) Chysanthemum. In: Nagata T, Lorz H, Widholm JM (eds) Biotechnology in Agriculture and Forestry Vol 61. Springer, Berlin Heidelberg New York, pp 253-272 